\title{
Morte Encefálica: Conhecimento e Opinião dos Médicos da Unidade de Terapia Intensiva
}

\author{
Brain Death: Knowledge and Opinion of \\ Physicians from an Intensive Care Unit
}

Daniel Ribeiro Soares de SouzaI Priscilla Passarelli Tostes ${ }^{I}$ Alexandre Sousa Silva ${ }^{I I}$

\section{PALAVRAS-CHAVE}

- Terapia Intensiva.

- Morte Encefálica.

- Obtenção de Órgãos e Tecidos.
${ }^{I}$ Universidade Federal do Rio de Janeiro, Rio de Janeiro, Rio de Janeiro, Brasil.

${ }^{I I}$ Universidade Federal do Estado do Rio de Janeiro, Rio de Janeiro, Rio de Janeiro, Brasil.
Objetivos: Verificar o conhecimento dos médicos de UTI sobre o diagnóstico de morte encefálica $(M E)$ e averiguar a opinião dos médicos de UTI sobre doação de órgãos. Métodos: Estudo quantitativo, exploratório, descritivo e transversal. Foram entrevistados 38 médicos que trabalham em UTI adulto em um hospital estadual da cidade do Rio de Janeiro. O instrumento de coleta de dados foi dividido em três partes: a primeira se referia a informações profissionais; a segunda era composta por nove questões fechadas, de múltipla escolha, que abordavam critérios técnicos para a realização do diagnóstico de morte encefálica; outras sete questionavam a opinião dos entrevistados sobre morte encefálica e doação de órgãos. Resultados: A população do estudo possui média de idade de 38,45 anos (DP = 10,58). Em relação ao tempo de formação, observou-se a média de 11,87 anos $(D P=8,94)$. O quantitativo geral de acertos das questões conceituais foi de 8,07 ( $D P=0,78)$. Dos 38 participantes, apenas $31,57 \%$ acertaram todas as questões. Observou-se que dois entrevistados (5,2\%) não se consideravam seguros em realizar o exame clínico. Não foram encontradas diferenças significativas nos números de acertos em comparações referentes a idade e/ou sexo dos entrevistados. Conclusão: Somente o grupo profissional de intensivistas teve participantes que acertaram todas as questões técnicas. No entanto, algumas questões básicas precisam ser mais bem discutidas. É importante a incorporação de disciplinas que abordem o tema nos cursos de graduação da área de saúde. Atitudes educativas sobre o tema podem ser mais bem difundidas nos cursos de graduação das diversas áreas de saúde, com a inclusão de disciplinas na grade curricular, bem como a abordagem do tema de forma extensiva nos cursos de especialização em terapia intensiva, de modo a permitir que se formem profissionais com maior grau de conhecimento sobre todo o contex to que envolve a ME e o processo doação-transplante, uma vez que esse processo não admite falhas em nenhuma das etapas. 


\section{KEY-WORDS}

- Intensive Care.

- Brain Death

- Organs and Tissue

Procurement.

\begin{abstract}
Objectives: Verify the knowledge of ICU physicians about the diagnosis of brain death and the opinions of ICU physicians about organ donation. Methods: Quantitative, exploratory, descriptive and cross-sectional study. Methods: We interviewed 38 physicians at an adult ICU of a State hospital in the city of Rio de Janeiro. The data collection instrument was split into three parts: the first to gather professional information; the second was composed of nine closed-ended, multiple-choice questions, which addressed technical criteria for the diagnosis of brain death; a further seven questions asked for the interviewee's opinion on brain death and organ donation. Results: The study population had a mean age of 38.45 years $(D P=10.58)$. In relation to training time, the average was 11.87 years (DP = 8.94). The overall quantity of correct answers to the conceptual questions was $8.07(D P=0.78)$. Of the 38 participants, only $12(31.57 \%)$ correctly answered all the questions. Two interviewees $(5.2 \%)$ did not feel confident about conducting the clinical examination. No significant differences were found between the number of correct anwers and the age and/or gender of the interviewee. Conclusion: Only the professional group of intensivists had participants who answered all the technical questions correctly. However, some basic issues require deeper discussion. It is important to incorporate disciplines that approach this subject in undergraduate courses in the health area, which may help diffuse educational attitudes. Such themes should also be extensively addressed in specialization courses in intensive care in order to improve training about the whole context involving brain death and the donation-transplant process, since this process must be absolutely watertight at every step.
\end{abstract}

Recebido em: 3/3/19

Aceito em: 3/3/19

\section{INTRODUÇÃO}

A morte encefálica (ME) pode ser definida como estado irreversível de cessação de todo o encéfalo e funções neurais, considerando tanto os hemisférios cerebrais como o tronco encefálico, resultante de edema e maciça destruição dos tecidos encefálicos, apesar de a função cardiopulmonar poder ser mantida por meio de sistema avançado de suporte vital e mecanismos de ventilação ${ }^{1,2}$.

As principais causas que levam à ME são traumatismo cranioencefálico, hemorragias subaracnoideas, lesão difusa do cérebro após parada cardiorrespiratória revertida, hemorragia cerebral espontânea maciça, lesões isquêmicas grandes, meningoencefalites e encefalites fulminantes e falência hepática aguda por hepatite viral ou tóxica ou Síndrome de Reye ${ }^{3}$.

Do total de óbitos de um hospital, estima-se que de 1\% a $4 \%$ sejam por ME. Em unidades de terapia intensiva, estes valores se elevam para $10-15 \%$. Portanto, é de extrema importância o conhecimento dos profissionais médicos sobre as questões referentes ao diagnóstico de ME, uma vez que a identificação dos casos é o primeiro passo de um minucioso processo quando se vislumbra a possibilidade de doação de órgãos ${ }^{4}$.
O diagnóstico de morte encefálica é complexo, demandando uma série de fatores iniciais como pré-requisitos, tais como: conhecimento da causa do coma, ausência de hipotermia e ausência de ação de medicamentos depressores do sistema nervoso central (SNC) ${ }^{5}$.

Posteriormente são avaliadas três bases clínicas para constatação do óbito: coma sem resposta, ausência de reflexos de tronco encefálico e apneia. Não havendo contraindicações, o paciente é considerado um potencial doador de órgãos (PD) e poderá se tornar um doador efetivo após autorização familiar $^{5,6}$.

Um estudo realizado no Rio do Grande do Norte identificou diversos fatores que contribuem para a não efetivação de doadores, entre eles o desconhecimento do conceito de $\mathrm{ME}$, tanto pela população quanto pelos profissionais da saúde. Uma pesquisa similar apontou que o conhecimento dos profissionais sobre diagnóstico de ME e manutenção do PD é insuficiente ${ }^{6,7}$.

Com base no exposto e diante da percepção frente às dificuldades em realizar o protocolo de $\mathrm{ME}$, surgiram inquietações e interesse em discutir mais a fundo as questões relativas ao conhecimento sobre o assunto. A equipe médica é o sujeito 
principal do estudo proposto, uma vez que participa de todas as etapas envolvidas no processo de morte encefálica e manutenção do potencial doador de órgãos.

\section{OBJETIVOS}

Verificar o conhecimento dos médicos atuantes em uma UTI sobre o diagnóstico de ME e averiguar a opinião desses médicos sobre doação de órgãos.

\section{MATERIAIS E MÉTODOS}

Foi realizado um estudo quantitativo, exploratório, descritivo, transversal, aprovado pelo CEP do Instituto Israelita de Ensino e Pesquisa Albert Einstein (CAAE:19024013.70000.0071), no qual foram avaliados 38 médicos que trabalham em UTI para adultos em um hospital estadual da cidade do Rio de Janeiro. A coleta de dados foi realizada de outubro de 2013 a janeiro de 2014, durante o horário de trabalho na UTI. Na entrevista, foi explicado o objetivo da pesquisa, e, após a assinatura do Termo de Consentimento Livre e Esclarecido, os pesquisadores apresentaram o instrumento de pesquisa (Apêndice) e aguardaram a resposta daqueles que concordaram em participar.

$\mathrm{O}$ instrumento de coleta de dados foi dividido em três partes, sendo a primeira referente a informações profissionais, tais como tempo de formação, especialidade e número de participações em protocolos de morte encefálica. A segunda parte era composta de nove questões fechadas, de múltipla escolha, sobre critérios técnicos para a realização do diagnóstico de morte encefálica. As questões técnicas relativas ao protocolo de ME foram baseadas nas Resoluções $n^{\circ} 1.480 / 97$ e $n^{\circ}$ 1.826/07 do CFM, que definem os critérios para o diagnóstico de ME no Brasil, a aplicabilidade dos mesmos e a suspensão do suporte terapêutico após conclusão do diagnóstico. Outras sete questionavam a opinião dos entrevistados sobre morte encefálica e doação de órgãos.

Os dados foram categorizados e tabulados em planilha eletrônica no software Microsoft Excel 2016® e, em seguida, analisados no programa estatístico R (Rversion 3.0.2@2013), cujos resultados foram apresentados em tabelas e gráficos. Para análise de associações estatísticas, foram utilizados o teste qui-quadrado e o teste de Kruskal-Wallis. O objetivo destas análises foi encontrar associações estatísticas entre o número de acertos nas questões técnicas do instrumento em relação a especialidade, idade, sexo, tempo de formação e/ou número de participações prévias em protocolos de morte encefálica.

\section{RESULTADOS}

O hospital onde a pesquisa foi realizada é considerado de grande porte, com serviço de neurocirurgia e um total de 341 leitos, sendo 38 leitos de UTI adulta, além de 21 leitos extras com suporte intensivo (emergência adulta, pediatria e unidade pós-operatória).

No ano de 2013, o número total de óbitos foi de 2.169, sendo notificados apenas 81 casos de ME, o que representa 3,7\% do número total de óbitos, abaixo da estimativa prevista em literatura ${ }^{4}$.

A população do estudo (Tabela 1) possui média de idade de 38,45 anos (DP = 10,58). Em relação ao tempo de formação, observou-se uma média de 11,87 anos (DP = 8,94).

Foram entrevistados 38 médicos, que possuem especialização em terapia intensiva e/ou Clínica Médica, Cirurgia Geral, Cardiologia e Anestesiologia. Para fins estatísticos, os grupos de especialidades foram divididos entre intensivistas e não intensivistas, sendo este último composto por profissionais sem qualificação comprovada em terapia intensiva e/ou pertencentes a outras especialidades médicas.

O quantitativo geral de acertos das questões conceituais foi de 8,07 ( $\mathrm{DP}=0,78)$. Dos 38 participantes, apenas 12 $(31,57 \%)$ acertaram todas as questões.

Entre os participantes, observou-se que dois entrevistados $(5,2 \%)$ não se consideravam seguros em realizar o exame clíni$\mathrm{co}$, sendo caracterizados como não intensivistas, pertencentes ao sexo feminino, com idades e tempo de formação discrepantes. Ressalta-se também a não participação prévia em testes diagnósticos de ME destas entrevistadas.

Observando-se o padrão de respostas dos entrevistados, as questões com maior índice de acertos se referiam aos critérios de inviabilidade da abertura do protocolo de ME, elementos básicos para realização dos testes de morte encefálica e situações de exclusão para a doação, onde todos os participantes obtiveram 100\% de acertos (Gráfico 1).

Um nível de erros intermediário foi encontrado em três elementos básicos do diagnóstico de ME: os exames complementares válidos, assim como o horário correto do óbito, ambos com 10,52\% de erros. O intervalo de tempo necessário entre os exames apresentou $15,78 \%$ de respostas incorretas.

A descrição do fluxograma correto de atendimento ao potencial doador de órgãos obteve a menor quantidade de acertos: $36,82 \%$ dos participantes não atingiram o objetivo proposto.

Somente o grupo profissional de intensivistas teve participantes que acertaram todas as questões técnicas (Gráfico 2), embora não tenha sido encontrada associação estatística (p-valor $=0,1201)$. Quanto à participação ou execução de um protocolo de diagnóstico de morte encefálica, 2 participantes afirmaram nunca terem participado de nenhum protocolo; 7 $(18,4 \%)$ entrevistados participaram de 1 a 5 exames durante 


\begin{tabular}{|c|c|c|c|c|c|}
\hline & & descriti & ss entrev & & \\
\hline Variável & Média de Acertos & & urança no & & $\mathrm{n}$ \\
\hline & (DP) & Sim $(\%)$ & Não (\%) & (p-valor)* & $(\%)$ \\
\hline Idade anos & & & & 0,658 & \\
\hline $25-30$ & $7,8(0,774)$ & $14(93,3)$ & $1(6,7)$ & & $15(39,47)$ \\
\hline $31-40$ & $8,5(0,534)$ & $8(100,0)$ & 0 & & $8(21,05)$ \\
\hline $41-50$ & $8,2(0,487)$ & $6(85,7)$ & $1(14,3)$ & & $7(18,42)$ \\
\hline 50 ou mais & $8,0(1,069)$ & $8(100,0)$ & 0 & & $8(21,05)$ \\
\hline Sexo & & & & 0,149 & \\
\hline Masculino & $8,0(0,703)$ & $23(100,0)$ & 0 & & $23(60,53)$ \\
\hline Feminino & $8,0(0,848)$ & $13(86,7)$ & $2(13,3)$ & & $15(39,47)$ \\
\hline Tempo de formação & & & & 1,000 & \\
\hline $1-5$ & $7,8(0,806)$ & $15(93,8)$ & $1(6,2)$ & & $16(42,11)$ \\
\hline $6-10$ & $8,5(0,577)$ & $4(100,0)$ & 0 & & $4(10,53)$ \\
\hline $11-20$ & $8,2(0,646)$ & $10(90,9)$ & $1(9,1)$ & & $11(28,95)$ \\
\hline 21 ou mais & $8,0(1,000)$ & $7(100,0)$ & 0 & & $7(18,42)$ \\
\hline Especialidade & & & & 0,193 & \\
\hline Intensivistas & $8,2(0,768)$ & $21(100,0)$ & 0 & & $21(55,26)$ \\
\hline Não-intensivistas & $7,8(0,781)$ & $15(88,2)$ & $2(11,8)$ & & $17(44,74)$ \\
\hline Total & & & & & $38(100,00)$ \\
\hline
\end{tabular}

$D P=$ desvio padrão; $E C=$ exame clínico $;{ }^{*}$ teste exato de Fisher

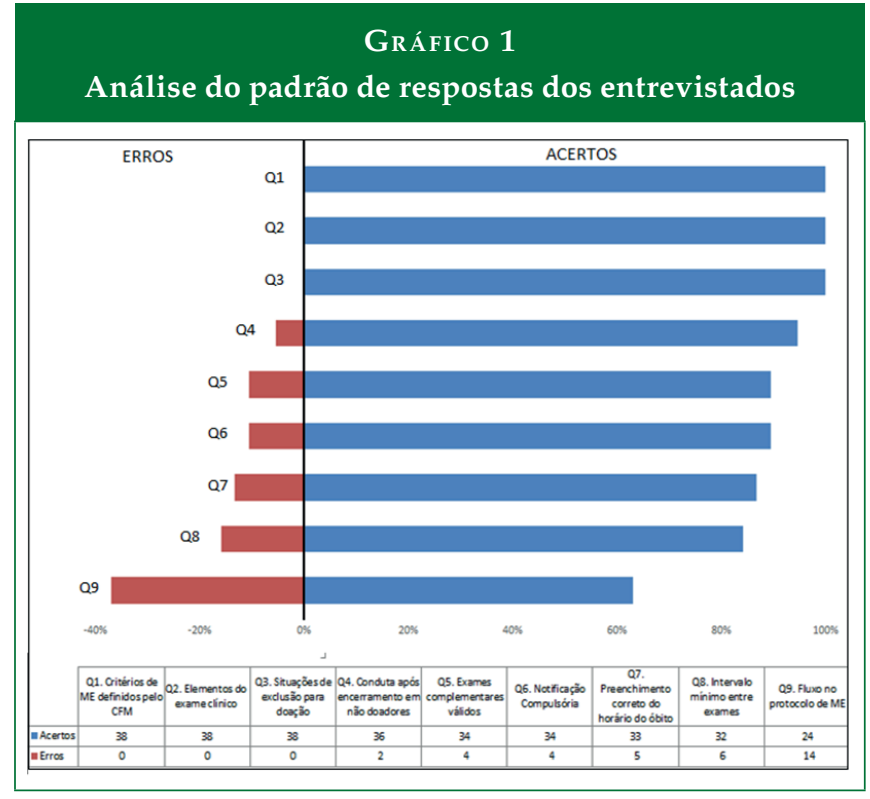

sua vida profissional; e 10 (26,3\%) e 19 (50\%) médicos participaram de 5 a 10 exames e de mais de 10 protocolos, respectivamente. Entretanto, a participação prévia em exame não está relacionada a maior índice de acertos da pesquisa ( $\mathrm{p}$-valor = 0,725; Gráfico 3).

Estratificando os acertos pelo tempo de formação, observou-se que o grupo de maior pontuação tinha de seis a dez

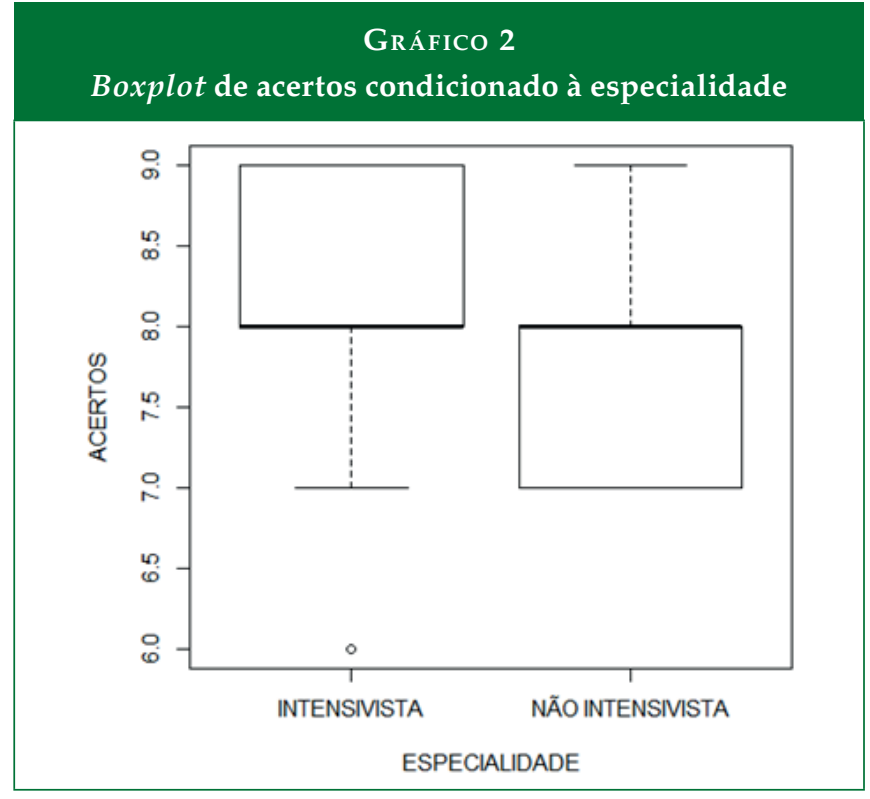

anos de formação (média $=8,5$ acertos; $\mathrm{DP}=0,57$ ), enquanto o grupo de menor quantitativo de acertos possuía de um a cinco anos de formação (média =7,8; DP = 0,80) (Gráfico 4).

Não foram encontradas diferenças significativas nos números de acertos em comparações referentes a idade e/ou sexo dos entrevistados. 

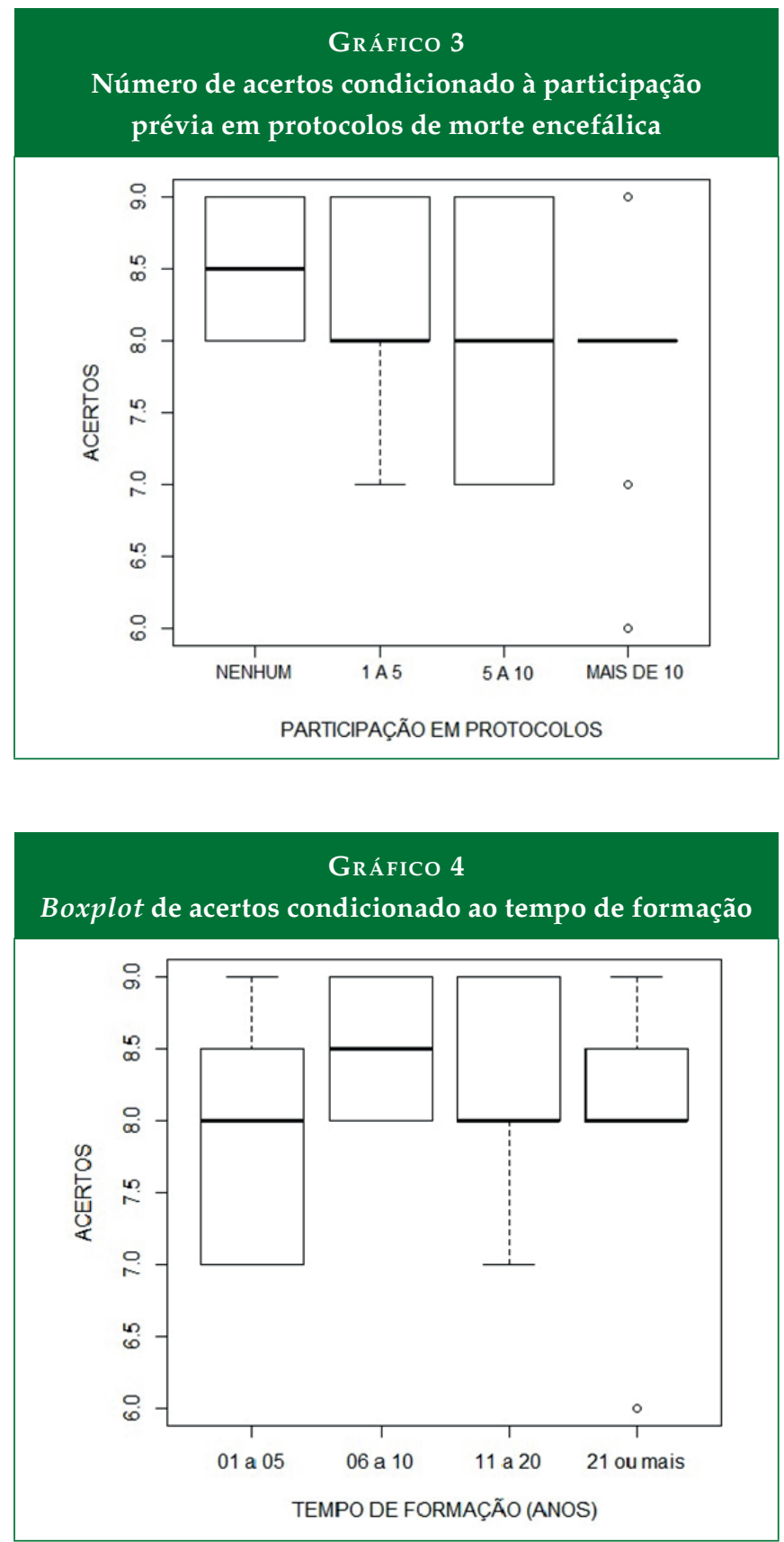

\section{DISCUSSÃO}

O presente estudo tentou avaliar o conhecimento e opinião de médicos de UTI sobre morte encefálica. Espera-se dos médicos intensivistas o mais alto nível de conhecimento a respeito da doação de órgãos e dos critérios de $\mathrm{ME}^{9}$.

Os resultados obtidos demonstram que os médicos, em geral, possuem uma média de acertos de $80 \%$. Um estudo similar na Coreia do Sul observou que profissionais apresentam falhas no entendimento do processo, também em pequenos índices ${ }^{10}$. No entanto, no que diz respeito ao intervalo mínimo da realização entre os dois exames clínicos e tipos de exames complementares aptos para execução do protocolo, os resultados merecem atenção por se tratar de elementos fundamentais no processo diagnóstico.

Embora sem comprovação estatística, observou-se menor número de acertos nos participantes com formação mais recente. Um estudo anterior que analisou o conhecimento de estudantes de Medicina demonstrou baixo conhecimento durante a vida acadêmica ${ }^{11}$.

Verificou-se também que alguns médicos não souberam definir o momento exato da constatação do óbito. A data e hora da declaração de óbito devem ser as mesmas em que foi constatada a ME, considerando para isto a do último exame do protocolo, seja clínico ou complementar ${ }^{12}$. Em um estudo anterior sobre conhecimento de médicos sobre ME, 71\% determinaram corretamente a hora do óbito. Entretanto, 24\% dos entrevistados consideraram como hora correta o momento da retirada dos órgãos para doação, o que vai contra o princípio ético e legal ${ }^{13}$. Isso demonstra a necessidade de investir na capacitação profissional para esse diagnóstico.

Apenas dois entrevistados não se consideravam seguros para realizar o exame clínico. Mesmo compondo um pequeno número, esse dado precisa ser bem avaliado e aprimorado, uma vez que a UTI é o local com maior quantitativo de pacientes com ME, sendo adequado que todos os profissionais médicos que ali trabalham estejam aptos. Outro dado de destaque é a margem de erros encontrada nos questionários de profissionais com experiência prévia no exame diagnóstico. Uma abordagem educacional pode se mostrar favorável à redução desses índices de insegurança e desconhecimento, já que pesquisadores alemães e portugueses demonstraram que treinamentos prévios contribuem para uma melhora no desempenho referente ao tema ${ }^{14,15,16}$.

Os resultados apontaram um número alto $(94,8 \%)$ de médicos que consideraram ética e legal a suspensão do suporte terapêutico, mas nem todos se sentem seguros para fazê-lo ou possuem opinião definida. Uma pesquisa realizada com profissionais de saúde de diferentes especializações indicou que $100 \%$ dos entrevistados aceitavam o conceito de ME, porém apenas $44 \%$ desligariam o suporte após o diagnóstico ${ }^{17}$. Esses posicionamentos podem ter como base a formação acadêmica, já que, em um estudo sobre conhecimento de morte encefálica em estudantes de Medicina no Brasil, 100\% afirmaram que esse tema não foi abordado na disciplina de Bioética, deixando os profissionais vulneráveis nesse âmbito de discussão ${ }^{18}$.

Sobre incentivarem a doação de órgãos e se considerarem doadores, o resultado foi bastante positivo e relevante, princi- 
palmente por se tratar de uma instituição que estimula a doação de órgãos. Pesquisas anteriores identificaram associações positivas ao incentivo à doação quando a equipe assistente tem comportamento favorável ${ }^{19}$.

\section{CONCLUSÃO}

Ainda são insuficientes os estudos que avaliam o conhecimento de médicos intensivistas sobre o assunto e fica evidente a necessidade de novas pesquisas para compreensão e interpretação dos dados obtidos.

Considera-se extremamente importante que as atitudes educativas sobre o tema sejam mais bem difundidas nos cursos de graduação das diversas áreas de saúde, com a inclusão de disciplinas na grade curricular e a abordagem extensiva do tema nos cursos de especialização em terapia intensiva, de modo a permitir que se formem profissionais com maior grau de conhecimento sobre todo o contexto que envolve a ME e o processo doação-transplante.

Além disso, é necessário que todos os profissionais de terapia intensiva se familiarizem com o assunto e tenham pleno domínio sobre ele, uma vez que se trata de um tema em que não há margem para erros. Para isso, sugerem-se treinamento e capacitação constantes dos profissionais. Objetiva-se, desta forma, um aumento do número de diagnósticos, condutas médicas mais uniformes e, consequentemente, a elevação dos índices de doações de órgãos.

\section{REFERÊNCIAS}

1. Brasil. Conselho Federal de Medicina. Resolução CFM n⿳ำ 1.480, de 8 de agosto de 1997. [Internet]. Dispõe sobre a caracterização de morte encefálica. Brasília: CFM; 1997 [acesso 02 junho 2017]. Disponível: http:/ / www.portalmedico. org.br/resolucoes/CFM/1997/1480_1997.htm.

2. Associação de Medicina Intensiva Brasileira. Morte Encefálica. Curso de imersão em terapia intensiva neurológica. $4^{\mathrm{a}}$ ed. São Paulo (SP): AMIB; 2005.

3. André C, Morte Cerebral - diagnóstico e suporte clínico. In: André C. Freitas GR. Terapia intensiva em neurologia e neurocirurgia - métodos de monitorização e situações especiais. Rio de Janeiro: Revinter; 2002. p. 303-23.

4. Garcia, VD. A política de Transplante no Brasil. Revista da AMRIGS [internet]. 2006 Out-Dez; 50(4):313-20. [acesso 2017 Jul 15]. Disponível em: http:/ / www.amrigs.org.br/ revista/50-04/aesp01.pdf.

5. Morato EG. Morte encefálica: conceitos essenciais, diagnóstico e atualização. Rev Med Minas Gerais [Internet]. [acesso 2018 Jan 10];19(3):227-36. Available from: http:/ / rmmg.org/artigo/detalhes/428
6. Lima CSP, Batista ACDO, Barbosa SDFF. Percepções da equipe de enfermagem no cuidado ao paciente em morte encefálica. Rev Eletrônica Enferm [Internet]. 2013;15(3):780-9. Available from: http://revistas.ufg.br/ index.php/fen/article/view/17497

7. Freire ILS, Mendonça AE de O, Pontes VO de, Vasconcelos QLD de AQ, Torre G de V. Morte encefálica e cuidados na manutenção do potencial doador de órgãos e tecidos para transplante. J Nurs UFPE Online [Internet]. 2012;14(4):90312. Available from: http://www.fen.ufg.br/revista/v14/ n4/pdf/v14n4a19.pdf

8. Bitencourt AGV, Neves FBCS, Durães L, Nascimento DT, Neves NMBC, Torreão L de A, et al. Avaliação do conhecimento de estudantes de medicina sobre morte encefálica. Rev Bras Ter Intensiva [Internet]. Revista Brasileira de Terapia Intensiva; 2007 Jun 1 [cited 2018 Jan 20];19(2):144-50. Available from: http://www.scielo.br/ scielo.php?script=sci_arttext\&pid=S0103-507X2007000200 002\&lng=pt\&nrm $=$ iso\&tlng $=$ pt

9. Brasil. Decreto-lei n. 2.268, de 30 de junho de 1997. Diário Oficial da União, Brasília 1 de jul. De 1997.

10. Jeon KO, Kim BN, Kim HS, Byeon N, Hong JJ, Bae SH, et al. A Study on Knowledge and Attitude toward Brain Death and Organ Retrieval among Health Care Professionals in Korea. Transplant Proc [Internet]. Elsevier Inc.; 2012;44(4):859-61. Available from: http://dx.doi. org/10.1016/j.transproceed.2012.02.019

11. Bitencourt AGV, Neves FBCS, Durães L, Nascimento DT, Neves NMBC, Torreão L de A, et al. Avaliação do conhecimento de estudantes de medicina sobre morte encefálica. Rev Bras Ter Intensiva [Internet]. Revista Brasileira de Terapia Intensiva; 2007 Jun 1 [cited 2018 Jan 20];19(2):144-50. Available from: http://www.scielo.br/ scielo.php?script=sci_arttext\&pid=S0103-507X2007000200 002\&lng $=$ pt\&nrm $=$ iso\&tlng $=$ pt

12. Brasil. Conselho Federal de Medicina. Resolução CFM n.1.826, de 06 de dezembro de 2007. [Internet]. Dispõe dobrea legalidade e o caráter ético da suspensão dos procedimentos de suportes terapêuticos quando da determinação de morte encefálica em indivíduo não-doador.. Brasília: CFM; 2007 [acesso em 2017 março 01]. Disponível em: http://www. portalmedico.org.br/resolucoes/cfm/2007/1826_2007.htm.

13. Schein A E, Carvalho P R A, Rocha TS, Guedes RR, Moschetti L, Salvia JC, Salvia PA - Avaliação do Conhecimento de Intensivistas sobre Morte Encefálica, RBTI 2008:20:2:144-148.

14. Radunz S, Hertel S, Schmid KW, Heuer M, Stommel P, Frühauf NR, et al. Attitude of Health Care Professionals to 
Organ Donation : Two Surveys Among the Staff of a German University Hospital. Transplant Proc [Internet]. Elsevier Inc.; 2010;42(1):126-9. Available from: http:/ /dx.doi. org/10.1016/j.transproceed.2009.12.034

15. Melo J, Batista A, Teixeira A, Figueiredo E, Ribeiro O, Lopes $\mathrm{P}$, et al. Knowledge and Behavior Among Health Professionals in Relation to Cadaveric Organ Donation and Transplantation : A Questionnaire- Based Analysis in Portuguese Hospitals. TPS [Internet]. Elsevier Inc.; 2011;43(5):142933. Available from: http://dx.doi.org/10.1016/j.transproceed.2010.11.028

16. Bilgin N, Akgun HS. A Focus on Health Care Professionals in Organ Donation : A Cross-Sectional Survey. Transplant Proc. 2002;1345(77):2445-7.
17. Rowinski W, Ostrowski K, Adadynski L et al - Factors limiting renal transplantation program in Poland. Ann Transplant, 1996;1:18-22. [acesso em 2017 junho 28]. Disponível em: http:/ /bases.bireme.br/cgi-bin/wxislind.exe/iah/online/

18. Dibo FHA, Gravena ÂAF, Freitas RA De, Agnolo CMD, Benguella EDA, Pelloso SM, et al. Brain Death: Knowledge of Future Brazilian Physicians. Transplant Proc [Internet]. Elsevier Inc.; 2017;49(4):750-5. Available from: http:/ /dx.doi.org/10.1016/j.transproceed.2017.01.069

19. Araujo C, Siqueira M. Brazilian Healthcare Professionals: A Study of Attitudes Toward Organ Donation. Transplant Proc [Internet]. Elsevier Inc.; 2016;48(10):3241-4. Available from: http://dx.doi.org/10.1016/j.transproceed.2016.09.044

\section{APÊNDICE}

Instrumento de Pesquisa

Data de nascimento: Ano de Formação: Gênero: ( ) Fem. ( ) Masc.

Especialidade:

Instituição:

1. De quantos protocolos de Morte Encefálica (ME) você já participou?
a) Nenhum
b) De 1 a 5
c) De 5 a 10
d) Mais de 10

2. Assinale abaixo quais situações devem ser excluídas para a abertura do protocolo de diagnóstico de ME:
a) Hipertermia / Hipertensão / Anúria / Drogas de- pressoras de SNC
b) Uso de drogas vasoativas / Hipertermia / Hipergli- cemia / Poliúria
c) Uso de drogas depressoras de SNC / Hipotermia / Causa do coma desconhecida / Instabilidade hemo- dinâmica

3. Para realizar prova clínica para diagnosticar o paciente em ME é necessário realizar exames clínicos neurológicos. Assinale os exames que fazem parte dos elementos do exame neurológico:

a) Coma aperceptivo / Reflexo medular / Reflexo cutâneo-plantar / Apneia / Ausência de reflexo de tosse

b) Coma aperceptivo / Ausência do reflexo córneo-palpebral / Ausência do reflexo oculocefálico / Ausência de resposta às provas calóricas / Ausência de reflexo de tosse / Apneia c) Coma aperceptivo / Ausência de reflexos flexores de retirada de MMII / Reflexo de Lázaro / Apneia / Ausência de reflexo de tosse

4. Coube ao CFM, através de suas resoluções (1480/97 e 1826/07), definir os critérios para determinação de ME e a aplicabilidade dos mesmos. Portanto, após seu diagnóstico, cabe ao médico preencher a Declaração de Óbito, caso esse não tenha sido ocasionado por meio violento. Ao preencher a data e hora do óbito, o que deve ser registrado?
a) Data e hora da abertura do protocolo de $\mathrm{ME}$
b) Data e hora da $2^{a}$ prova clínica para $\mathrm{ME}$
c) Data e hora do último exame do protocolo de $\mathrm{ME}$
d) Hora da parada cardiorrespiratória

5. De acordo com a resolução do CFM 1.826/07, após o encerramento do protocolo de $\mathrm{ME}$, em pacientes não doadores de órgãos, é correto afirmar que:
a) É ética e legal a suspensão do suporte terapêutico
b) Deve-se manter o suporte terapêutico até que ocorra a PCR
c) Não se pode suspender o suporte terapêutico, pois caracteriza eutanásia
d) É ética e legal a suspensão do suporte terapêutico e deve ser precedida de comunicação e esclarecimento sobre a morte encefálica aos familiares do paciente ou seu repre- sentante legal, fundamentada e registrada no prontuário


6. O diagnóstico de $\mathrm{ME}$ determinado pela resolução 1.480/97 do CFM é realizado através de:

a) Dois exames clínicos, realizados por dois médicos diferentes, e não ligados à equipe transplantadora, e um exame complementar

b) Dois exames clínicos, sendo pelo menos um realizado pelo médico da Central de Transplantes, e dois exames complementares, sendo um deles a tomografia

c) Dois exames clínicos, realizados por dois médicos diferentes, sem a obrigatoriedade do exame complementar

7. Após o término do protocolo de ME, o que deve ser feito:

a) Finalizar o Termo de Declaração de ME, assinar o atestado de óbito ou termo de declaração para o IML, comunicar o caso ao órgão competente (SPOT/OPO/ Central de Transplantes) e informar o óbito aos familiares

b) Notificar à CIHDOTT, que será responsável pelo preenchimento da Declaração de Óbito e pela manutenção hemodinâmica do potencial doador

c) Notificar à CIHDOTT, comunicar ao órgão competente e deixar a cargo do mesmo o preenchimento da Declaração de Óbito após a extração dos órgãos

8. Qual o intervalo de tempo mínimo preconizado entre o primeiro e o segundo exames clínicos para ME?

a) 6 horas em adulto e variável de acordo com a faixa etária em crianças de até 2 anos de idade

b) 6 horas, independentemente da faixa etária

c) 12 horas, independentemente da faixa etária

d) 24 horas, independentemente da faixa etária

9. Quais são os exames complementares para realização do diagnóstico de ME preconizados pelo CFM?
a) Arteriografia
b) EEG
c) Doppler
d) Todos acima

10. É importante a realização do protocolo de ME do paciente que preencher os critérios do mesmo e a notificação ao órgão competente, pois:
a) Ajuda a diminuir a fila de espera por um órgão
b) É uma notificação de caráter compulsório, com impli- cação legal se não realizada
c) É uma notificação de caráter compulsório, sem impli- cação legal se não realizada

11. A Resolução CFM $\mathbf{n}^{\circ} \mathbf{1 . 8 2 6 / 0 7}$ dispõe sobre a legalidade e o caráter ético da suspensão dos procedimentos terapêuticos quando da determinação da ME do indivíduo não doador. Qual a sua posição sobre o desligamento dos suportes clínicos?
a) Desligar comunicando a família
b) Não desligar
c) Não tenho posição definida
d) Desligar o suporte desde que a família tenha compre- endido o diagnóstico de $\mathrm{ME}$

12. Na sua opinião, o paciente que receber o diagnóstico de ME baseado nos critérios definidos pela resolução CFM 1.480/97 pode ter o quadro neurológico revertido?
a) $\mathrm{Sim}$
b) Não

13. Você se sente seguro para realizar o protocolo de ME?
a) $\operatorname{Sim}$
b) Não

14. Se você precisasse de um transplante, aceitaria receber um órgão?
a) Sim
b) Não

15. Você incentivaria a doação de órgãos entre pessoas de suas relações?
a) $\operatorname{Sim}$
b) Não
c) Não tenho opinião

16. Você é doador de órgãos?
a) $\mathrm{Sim}$
b) Não

\section{CONTRIBUIÇÃO DOS AUTORES}

A autora Priscilla Passarelli Tostes realizou a elaboração do projeto e composição da revisão bibliográfica. O autor Daniel Ribeiro Soares de Souza aplicou os testes estatísticos, análise de dados e discussão. Alexandre Sousa Silva foi orientador e responsável pela revisão estatística e do texto.

\section{CONFLITO DE INTERESSES}

Não há.

\section{ENDEREÇO PARA CORRESPONDÊNCIA}

Daniel Ribeiro Soares de Souza

R. Prof. Rodolpho Paulo Rocco, 255, 7ํ․ Andar, Serviço de

Nefrologia - Ilha do Fundão, Rio de Janeiro - RJ,

CEP: 21941-590

Email: daniel.ribeiro@ufrj.br 